\title{
N49: THE FIRST ROBUST DISCOVERY OF RECOMBINING PLASMA IN AN EXTRA GALACTIC SUPERNOVA REMNANT
}

\section{$\operatorname{AUTHOR}(\mathrm{S}):$}

Uchida, Hiroyuki; Koyama, Katsuji; Yamaguchi, Hiroya

\section{CITATION:}

Uchida, Hiroyuki ...[et al]. N49: THE FIRST ROBUST DISCOVERY OF RECOMBINING PLASMA IN AN EXTRA GALACTIC SUPERNOVA REMNANT. The Astrophysical Journal 2015, 808(1):

77.

\section{ISSUE DATE:}

2015-07-21

URL:

http://hdl.handle.net/2433/202059

RIGHT:

(C) 2015. The American Astronomical Society. 


\title{
N49: THE FIRST ROBUST DISCOVERY OF RECOMBINING PLASMA IN AN EXTRA GALACTIC SUPERNOVA REMNANT
}

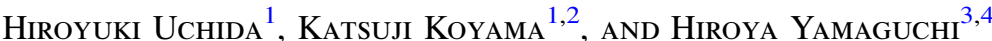 \\ ${ }^{1}$ Department of Physics, Graduate School of Science, Kyoto University, Kitashirakawa Oiwake-cho, Sakyo-ku, \\ Kyoto 606-8502, Japan; uchida@cr.scphys.kyoto-u.ac.jp \\ ${ }^{2}$ Department of Earth and Space Science, Graduate School of Science, Osaka University, 1-1 Machikaneyama, Toyonaka, Osaka 560-0043, Japan \\ ${ }^{3}$ NASA Goddard Space Flight Center, Code 662, Greenbelt, MD 20771, USA \\ ${ }^{4}$ Department of Astronomy, University of Maryland, College Park, MD 20742, USA \\ Received 2015 April 24; accepted 2015 May 28; published 2015 July 21
}

\begin{abstract}
Recent discoveries of recombining plasmas (RPs) in supernova remnants (SNRs) have dramatically changed our understanding of SNR evolution. To date, a dozen RP SNRs have been identified in the Galaxy. Here, we present Suzaku deep observations of four SNRs in the LMC, namely, N49, N49B, N23, and DEM L71, for accurate determination of their plasma states. Our uniform analysis reveals that of these SNRs, only N49 is in the recombining state, which is the first robust discovery of an RP from an extra-galactic SNR. Given that RPs have only been identified in core-collapse SNRs, our results strongly suggest a massive star origin for this SNR. On the other hand, no clear evidence of an RP is confirmed in N23, for which detection of recombination lines and continua was previously claimed. Comparing the physical properties of the RP SNRs identified so far, we find that all of them can be categorized into the "mixed-morphology" class, interacting with surrounding molecular clouds. This might be a key to determining the formation mechanisms of RPs.
\end{abstract}

Key words: ISM: abundances - ISM: individual objects (DEM L71, N23, N49, N49B) - ISM: supernova remnants $\mathrm{X}$-rays: ISM

\section{INTRODUCTION}

X-ray observations of supernova remnants (SNRs) allow us to make accurate measurements of plasma conditions and elemental abundances in the supernova $(\mathrm{SN})$ ejecta, providing unique insights into the progenitor's evolution and explosion as well as the dynamical evolution of the remnants themselves. The hot plasmas in X-ray-emitting SNRs are commonly in a state of non-equilibrium ionization (NEI) where the ionization degrees of heavy elements are inconsistent with those expected for a collisional ionization equilibrium (CIE) plasma with a certain electron temperature (e.g., Masai 1984). It was widely believed for a long time that SNR plasma is always in the lowionization state, and slowly ionizing to achieve CIE (hereafter, ionizing plasma (IP)). In fact, recent X-ray observations with sensitive satellites, like Suzaku (Mitsuda et al. 2007), have confirmed that the immediate post-shock gas in young SNRs indeed consists of extremely low ionized atoms together with hot electrons (e.g., Uchida et al. 2013; Yamaguchi et al. 2014b).

However, earlier ASCA observations had suggested the presence of recombining plasma (RP; where the atoms are overionized compared to the observed electron temperature) in a couple of SNRs, IC 443 and W49B (Kawasaki et al. 2002, 2005). Conclusive evidence for the RPs was later revealed by Suzaku observations that discovered enhanced radiative recombination continua (RRCs) in the X-ray spectra of these remnants (Ozawa et al. 2009; Yamaguchi et al. 2009). These results were followed by observational studies of other SNRs, leading to a significant increase in the number of RP SNRs. To date, RPs have been discovered in a dozen SNRs, which means that the presence of RPs is no longer unusual or surprising.

The well-identified RP SNRs are all categorized into the socalled mixed-morphology (MM) class, defined by centrally filled thermal X-ray emission with a synchrotron radio shell (Rho \& Petre 1998). While more than 25\% of the X-raydetected Galactic SNRs are classified into this type (Jones et al. 1998), the physical process occuring to form such characteristic morphology is still unclear. Note that, theoretically, dense ambient materials play an important role in the dynamical evolutions of MM SNRs (e.g., White \& Long 1991; Petruk 2001). Notably, another prediction is that the formation of RPs can also be explained by the interaction between the SN ejecta and the dense materials surrounding the progenitor (e.g., Itoh \& Masai 1989). Therefore, the physical processes that create the MM and RP seem to be related to each other.

It is worth noting that most of the RP SNRs identified thus far were previously considered to have IP or nearly CIE plasma (e.g., W44; Uchida et al. 2012). This implies that there are still a number of SNRs for which the plasma state has been misclassified. Here, we present a uniform analysis of highquality data of MM SNRs (as well as typical shell-like SNRs simultaneously observed) in the Large Magellanic Cloud (LMC) obtained using the X-ray Imaging Spectrometer (XIS; Koyama et al. 2007) on board Suzaku, in order to search for RPs in these SNRs. The LMC is particularly suitable for such a systematic study because of its low foreground extinction (Dickey \& Lockman 1990) and its known distance of $50 \mathrm{kpc}$ (Feast 1999); we use this distance throughout the paper.

In Section 2, we outline our observations and data reduction procedures. The results of the spectral analysis of four LMC SNRs, namely, N49, N23, N49B, and DEM L71, are presented in Section 3 where we will also show the new discovery of an RP from N49. We discuss our results in Section 4, and finally provide conclusions and future prospects in Section 5. The errors quoted in text, tables, and figures are at the $90 \%$ confidence level unless otherwise stated. 
Table 1

Observation Logs

\begin{tabular}{lcccc}
\hline \hline Target & Obs. ID & Obs. Date & (R.A., decl.) $)_{\text {J2000 }}$ & Exposure \\
\hline N23 \& DEM L71 & 807008010 & 2012 Apr 4 & $(76.45,-67.96)$ & $102 \mathrm{ks}$ \\
N49 \& N49B & 807007010 & 2012 May 9 & $(81.50,-66.08)$ & $185 \mathrm{ks}$ \\
\hline
\end{tabular}

\section{OBSERVATIONS AND DATA REDUCTION}

We observed two regions in the LMC aimed at the MM SNRs N23 and N49 during the Suzaku Cycle 8 phase. Detailed information of the observations is summarized in Table 1 . In addition to the targeted sources, the nearby SNRs DEM L71 and N49B are detected in each field of view (FOV) of the XIS. We also analyzed their spectra for a comparison of the plasma states among the targets. Both DEM L71 and N49B are classified as standard shell-like SNRs (Hughes et al. 2003; Park et al. 2003b) from which an RP has never been observed to date.

We analyzed data from three active CCDs: one is backilluminated (XIS1) and the others are front-illuminated (FI; XIS0 and XIS3), although only the merged and averaged FI CCD spectra are shown in figures in the subsequent sections. We used HEAsoft tools version 6.11 for the data reduction. The uncleaned data were reprocessed using the calibration database released in 2011 September, and screened with the standard event selection criteria for cleaned events. The resulting effective exposures were 102 and $185 \mathrm{ks}$ for the observations of N23 (with DEM L71) and N49 (with N49B), respectively.

\section{ANALYSIS AND RESULTS}

In Figure 1, we show the $0.6-10.0 \mathrm{keV}$ images of the two observations after subtraction of non X-ray background (NXB) generated using xisnxbgen (Tawa et al. 2008). Since the diameters of the SNRs $(\lesssim 1$ '.5; e.g., N49B; Park et al. 2003b) are smaller than the angular resolution of the X-Ray Telescopes (the half-power diameter ranges from 1!.8 to $2 ! 3$ ), our analysis focuses on spectra from the entire SNRs. We extract the source spectra from the regions enclosed by the circles shown in Figure 1. The background spectra are taken from the entire FOV excluding the source regions as well as the CCD corners illuminated by the ${ }^{55} \mathrm{Fe}$ calibration sources.

Figure 2 compares the background-subtracted spectra of the four SNRs, showing that N49 is the brightest among them (with a count rate about $\sim 10$ times higher than the others). Due to the good energy resolution of the XIS, we clearly detect $\mathrm{K}$-shell emission lines of $\mathrm{Ar}, \mathrm{Ca}$, and $\mathrm{Fe}$ from this remnant for the first time. The spectrum of N49 exhibits strong $\mathrm{Ly} \alpha$ lines of $\mathrm{Mg}, \mathrm{Si}$, and $\mathrm{S}$, whereas there is little or no signal of these lines from the other remnants. This indicates that the heavy elements in N49 are more highly ionized than those in the others. Since our main goal is to identify an RP SNR(s), we first perform detailed spectral analysis of N49 (Section 3.1), followed by an analysis of the other remnants (Sections 3.2-3.4).

We use the SPEX software version 2.04.01 (Kaastra et al. 1996) for the spectral fitting by taking into account the detector and telescope responses (the so-called "redistribution matrix" and "ancillary response," respectively) generated by xisrmfgen and xissimarfgen (Ishisaki et al. 2007). The data around the neutral $\mathrm{Si}$ K-shell edge $(1.77-1.83 \mathrm{keV})$ are ignored because of the poor accuracy in the response function
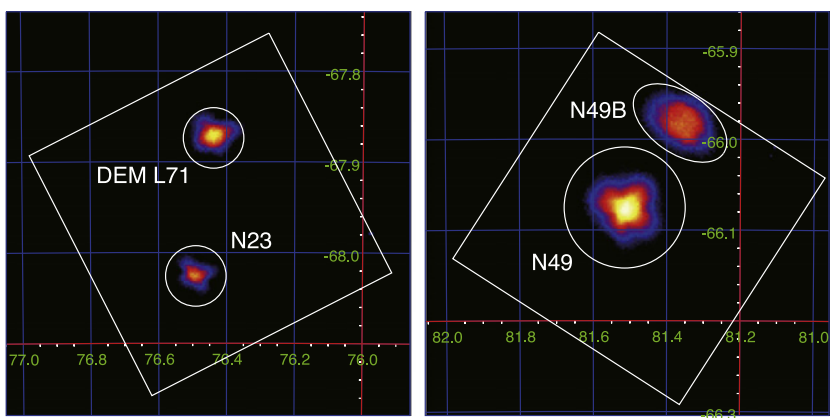

Figure 1. Vignetting-corrected XIS FI images of the N23 (left) and N49 (right) regions in the $0.6-10.0 \mathrm{keV}$ band with an equatorial coordinate grid. DEM L71 and N49B are also detected in the FOV of the XISs indicated by the solid squares.

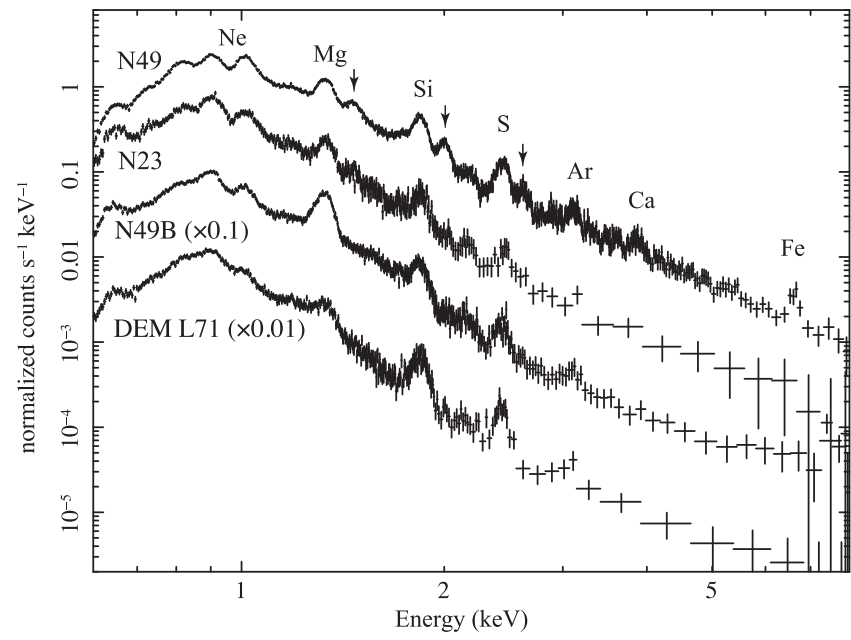

Figure 2. XIS FI spectra of N49, N23, N49B, and DEM L71 where the background spectra are subtracted. The spectra of N49B and DEM L71 are multiplied by factors of 0.1 and 0.01 , respectively. The arrows represent the line centroids of $\mathrm{Mg}-\mathrm{Ly} \alpha, \operatorname{Si}-\mathrm{Ly} \alpha$, and S-Ly $\alpha$.

at these energies. ${ }^{5}$ During the analysis, we separately set absorption column densities in the Milky Way $\left(N_{\mathrm{H}(\mathrm{MW})}\right)$ and the LMC $\left(N_{\mathrm{H}(\mathrm{LMC})}\right)$. The former value is fixed to $6 \times 10^{20} \mathrm{~cm}^{-2}$ (Dickey \& Lockman 1990). The elemental abundances of the latter component are fixed to the average values of the LMC ( 0.3 solar; Russell \& Dopita 1992).

\section{1. $N 49$}

N49 is one of the brightest SNRs in the LMC at the various wavelengths observed (e.g., Long et al. 1981; Dickel \& Milne 1998). The radio continuum and $\mathrm{H} \alpha$ emission exhibit a clear shell structure (Vancura et al. 1992; Dickel et al. 1995), whereas the X-ray image shows an irregular morphology that is brightest inside the radio shell in the southwest region (Park et al. 2012), as is a typical characteristic of an MM SNR. N49

http://heasarc.nasa.gov/docs/Suzaku/analysis/sical.html 


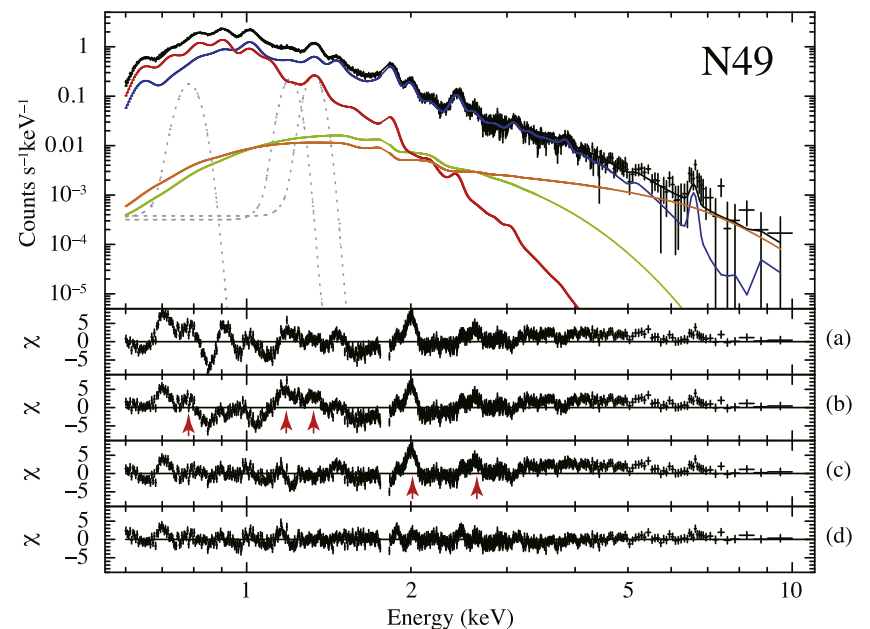

Figure 3. XIS FI spectrum of N49 (top panel). The best-fit model is overlaid with the solid black line. The solid blue and red lines represent the best-fit high- $k T_{\mathrm{e}}(\mathrm{RP})$ and the low- $k T_{\mathrm{e}}$ (IP) components, respectively. The orange and green lines show the power-law and the blackbody components for SGR 0526-66, respectively. The dotted lines show Gaussians for the Fe L emission missing from the plasma code we used. The residuals are shown in panel (d). Panels (a), (b), and (c) represent the residuals from the models of single IP, two IP, and two IP plus Gaussians, respectively.

spatially overlaps with the soft gamma-ray repeater (SGR) 0526-66 (Cline et al. 1982), although the physical association between the SGR and SNR is under debate (Gaensler et al. 2001; Badenes et al. 2009). Using Chandra data, Park et al. (2012) revealed that the X-ray spectrum of SGR 0526-66 is best reproduced by a model consisting of a blackbody (BB) with $k T_{\mathrm{BB}}=0.44 \pm 0.02 \mathrm{keV}$ and a power law (PL) with $\Gamma=2.50_{-0.12}^{+0.11}$. Unfortunately, our Suzaku data do not spatially resolve the SGR from the SNR. We thus add the best-fit model by Park et al. (2012) to the model spectrum of N49, but allow its spectral parameters to vary within the reported statistical uncertainty. Since Park et al. (2012) found significant longterm variability in the X-ray flux of the SGR, we also allow the fluxes of both BB and PL components to vary freely.

First, we apply a single-component IP model where the initial ionization balance is dominated by the neutral state for all heavy elements. The free parameters are the electron temperature $\left(k T_{\mathrm{e}}\right)$, ionization timescale $\left(n_{\mathrm{e}} t\right.$, where $n_{\mathrm{e}}$ and $t$ are the electron number density and the elapsed time since the gas was shock heated), emission measure $\left(\mathrm{EM}=\int n_{\mathrm{e}} n_{\mathrm{H}} V\right)$, and abundances of $\mathrm{O}, \mathrm{Ne}, \mathrm{Mg}, \mathrm{Si}, \mathrm{S}, \mathrm{Ar}, \mathrm{Ca}$, and $\mathrm{Fe}$ relative to the solar values of Anders \& Grevesse (1989). The Ni abundance is linked to the value of $\mathrm{Fe}$, while the abundances of the other elements are fixed to the LMC averages of Russell \& Dopita (1992). This model yields a best-fit electron temperature of $\sim 0.6 \mathrm{keV}$ but fails to reproduce the overall spectrum with an unacceptable $\chi^{2} /$ degrees of freedom (dof) of 4936/820. The residual is shown in panel (a) of Figure 3. The particularly large disagreement between the data and model is seen below $\sim 1 \mathrm{keV}$, implying the presence of another plasma component with a lower temperature. Assuming that this component originates from a swept-up interstellar medium (ISM) that is dominant at the outermost region of the remnant (Park et al. 2003a, 2012), we added another IP component with the abundances fixed to the LMC mean values. Although this twocomponent model gives a slightly better fit $\left(\chi^{2} /\right.$ dof $=4297 /$ $817)$, some line-like residuals still remain, as shown in panel (b) of Figure 3 . The residual at $\sim 0.8 \mathrm{keV}$, also observed in our previous studies of other objects (e.g., Nakashima et al. 2013; Uchida et al. 2013), is likely due to the well-known uncertainty in the emissivity ratio between the Fe XVIII L-shell emission of $3 s \rightarrow 2 p$ and $3 d \rightarrow 2 p$ (Gu et al. 2007). On the other hand, the residuals at $\sim 1.2$ and $\sim 1.3 \mathrm{keV}$ are caused by L-shell emission from high quantum numbers $(n>5)$ missing from the plasma code SPEX (Brickhouse et al. 2000). We compensate for these lines by adding three Gaussians at these energies and obtain the improved fit $\left(\chi^{2} / \mathrm{dof}=2366 / 815\right)$ with the best-fit $k T_{\mathrm{e}}$ of $\sim 0.6 \mathrm{keV}$ and $n_{\mathrm{e}} t$ of $\sim 10^{13} \mathrm{~cm}^{-3} \mathrm{~s}$ for the high- $k T_{\mathrm{e}}$ component. The obtained ionization timescale indicates that this IP model actually represents a CIE plasma at the given temperature.

The fit left the largest residuals at 2.0 and $2.6 \mathrm{keV}$ (see panel (c) of Figure 3), which correspond to the centroid energies of the Si Ly $\alpha$ and S Ly $\alpha$ emissions, respectively. This suggests that the average charge of these elements is higher than that expected in a $0.6 \mathrm{keV}$ CIE plasma. Furthermore, a hump-like feature is found in the residual around $2.7 \mathrm{keV}$, indicating an enhancement of the RRC of $\mathrm{Si}$, as was observed in the other RP SNRs (e.g., IC 443; Yamaguchi et al. 2009). Therefore, we introduce an RP model (using an "NEIJ" model in $\mathrm{SPEX}^{6}$ ) for the high- $k T_{\mathrm{e}}$ component. In addition to the parameters given in the IP model, the RP model has another free parameter $k T_{\text {init }}$ higher than $k T_{\mathrm{e}}$, which describes a recombining history by $n_{\mathrm{e}} t$ in the $k T_{\mathrm{e}}$ plasma starting from the initial ionization temperature of $k T_{\text {init }}$. This model dramatically improves the fit $\left(\chi^{2} / \mathrm{dof}=1270 / 813\right)$, removing the large residuals above $2 \mathrm{keV}$ as shown in the panel (d) of Figure 3. The best-fit model components and parameters are given in Figure 3 and Table 2, respectively.

Interestingly, the RP model reproduces the $\mathrm{Fe} \mathrm{K} \alpha$ emission at $\sim 6.6 \mathrm{keV}$ as well, although free electrons in the $0.6 \mathrm{keV}$ plasma are not energetic enough to excite K-shell electrons of Fe. This indicates that the observed $\mathrm{Fe} \mathrm{K} \alpha$ emission predominantly originates from cascade processes after radiative recombination into the excited levels of He-like ions (i.e., $\left.\mathrm{Fe}^{25+}+e^{-} \rightarrow \mathrm{Fe}^{24+*}\right)$. We emphasize that this is why $k T_{\text {init }}$ is constrained to the very high value $(\gtrsim 10 \mathrm{keV})$ so that a significant fraction of the Fe ions remains at the H-like state in the current plasma. It should also be noted that the observed centroid energy $6629_{-26}^{+31} \mathrm{eV}$ is consistent with the value expected from the best-fit RP model $(6658 \mathrm{eV})$ but significantly lower than that for a typical CIE plasma at $k T_{\mathrm{e}}=5-$ $10 \mathrm{keV}(\sim 6680 \mathrm{eV})$. This is another piece of evidence for the $\mathrm{RP}$; the forbidden and intercombination lines are likely to be enhanced by the recombination processes.

\section{2. $N 23$}

In the previous work using XMM-Newton MOS and RGS data, Broersen et al. (2011) claimed that the X-ray spectrum of N23 can be best reproduced by a two-component NEI model of which one of the components was an RP with $k T_{\text {init }}=3.0$ $\mathrm{keV}$ and $k T_{\mathrm{e}}=0.18 \mathrm{keV}$. We first fit the XIS spectrum fixing their best-fit parameters but fail to reproduce the XIS spectrum $\left(\chi^{2} /\right.$ dof $\left.=455 / 236\right)$, leaving large residuals around the Ne-Ly $\alpha$ emission and the Fe XVII recombination edge at $\sim 1.25 \mathrm{keV}$. The fit is not improved even if we allow the abundances to vary. Therefore, we thaw the temperatures and ionization parameters (but restricting $k T_{\text {init }}$ to be higher than $k T_{\mathrm{e}}$ ), obtaining a

6 See http://www.sron.nl/files/HEA/SPEX/manuals/manual.pdf for details. 
Table 2

Best-fit Parameters

\begin{tabular}{|c|c|c|c|c|c|}
\hline \multirow{2}{*}{ Component } & \multicolumn{5}{|l|}{ Parameter } \\
\hline & & N49 & $\mathrm{N} 23$ & $\mathrm{~N} 49 \mathrm{~B}$ & DEM L71 \\
\hline \multirow[t]{2}{*}{ Absorption } & $N_{\mathrm{H}(\mathrm{LMC})}\left(\times 10^{21} \mathrm{~cm}^{-2}\right)$ & $3.51_{-0.04}^{+0.01}$ & $2.13 \pm 0.05$ & $2.30_{-0.06}^{+0.01}$ & $3.70_{-0.50}^{+0.30}$ \\
\hline & $N_{\mathrm{H}(\mathrm{MW})}\left(\times 10^{21} \mathrm{~cm}^{-2}\right)$ & 0.6 (fixed) & 0.6 (fixed) & 0.6 (fixed) & 0.6 (fixed) \\
\hline \multirow[t]{3}{*}{ ISM } & $k T_{\mathrm{e}}(\mathrm{keV})$ & $0.30 \pm 0.01$ & $0.17 \pm 0.01$ & $0.21 \pm 0.01$ & $0.16 \pm 0.01$ \\
\hline & $n_{\mathrm{e}} t\left(\times 10^{11} \mathrm{~cm}^{-3} \mathrm{~s}\right)$ & $3.49_{-0.26}^{+0.09}$ & $>10$ & $1.23_{-0.07}^{+0.09}$ & $>10$ \\
\hline & $\mathrm{EM}\left(\times 10^{58} \mathrm{~cm}^{-3}\right)$ & $3.54_{-0.01}^{+0.03}$ & $17.6 \pm 0.3$ & $6.0 \pm 0.9$ & $19_{-4}^{+5}$ \\
\hline & $n_{\mathrm{e}} t\left(\times 10^{11} \mathrm{~cm}^{-3} \mathrm{~s}\right)$ & $7.00_{-0.02}^{+0.44}$ & $5.08_{-0.37}^{+0.42}$ & $0.84 \pm 0.01$ & $1.77 \pm 0.07$ \\
\hline & $\mathrm{O}$ & $1.52_{-0.03}^{+0.04}$ & $3.5 \pm 0.2$ & $0.63_{-0.02}^{+0.03}$ & $0.30_{-0.04}^{+0.05}$ \\
\hline & $\mathrm{Ne}$ & $0.98_{-0.02}^{+0.03}$ & $1.25 \pm 0.07$ & $0.45 \pm 0.01$ & $0.25 \pm 0.02$ \\
\hline & $\mathrm{Mg}$ & $0.82 \pm 0.02$ & $1.16 \pm 0.07$ & $1.24 \pm 0.02$ & $0.62 \pm 0.03$ \\
\hline & $\mathrm{Si}$ & $1.32_{-0.02}^{+0.05}$ & $1.2 \pm 0.4$ & $0.34 \pm 0.02$ & $0.48 \pm 0.04$ \\
\hline & $S$ & $1.46_{-0.11}^{+0.03}$ & $1.25 \pm 0.08$ & $0.50 \pm 0.08$ & $1.0 \pm 0.2$ \\
\hline & $\chi^{2} /$ dof & $1270 / 813$ & $218 / 234$ & $659 / 423$ & $291 / 229$ \\
\hline
\end{tabular}

significantly better fit with $\chi^{2} /$ dof $=224 / 234$ and $n_{\mathrm{e}} t>10^{12}$ $\mathrm{cm}^{-3} \mathrm{~s}$ for both components. This high $n_{\mathrm{e}} t$ value indicates that the plasmas are in nearly CIE independent of the initial ionization population (otherwise ionizing). In fact, we are not able to constrain $k T_{\text {init }}$ with this model. Finally, we apply an IP model consisting of an LMC-abundance component (for ISM) and a free-abundance component (for ejecta), and obtain a slightly better fit $\left(\chi^{2} /\right.$ dof $\left.=218 / 234\right)$ than the RP (or CIE) model. The best-fit parameters and models are given in Table 2 and Figure 4, respectively. During the analysis, we fix the spectral shape and flux of the faint X-ray compact source CXOU J050552.3-680141 associated with N23 to the reported values of Hayato et al. (2006), although its contribution is negligible at $\lesssim 3 \mathrm{keV}$ (see Figure 4 ).

\section{3. $N 49 B$}

N49B is a typical shell-like SNR containing no X-ray pointlike source within the SNR shell (Park et al. 2003b). We fit the XIS spectrum with the same two-component IP model applied to $\mathrm{N} 49$, obtaining the best-fit results given in Table 2 . The $n_{\mathrm{e}} t$ value for the ejecta component $\left(\sim 8 \times 10^{10} \mathrm{~cm}^{-3} \mathrm{~s}\right)$ is the lowest among the SNRs studied in this work. We reveal that the abundance of $\mathrm{Mg}$ is significantly higher than those of the other elements (e.g., $\mathrm{Mg} / \mathrm{O} \sim 2.0, \mathrm{Mg} / \mathrm{Ne} \sim 2.8, \mathrm{Mg} / \mathrm{Si} \sim 3.6$ ). Park et al. (2003b) previously found "Mg-rich" ejecta near the center of the remnant. Our result shows that the $\mathrm{Mg}$ enrichment is confirmed even in the integrated spectrum from the entire $\mathrm{SNR}$. We find no evidence for an RP; if $k T_{\text {init }}>k T_{\mathrm{e}}$ is assumed, then the model clearly fails to reproduce the spectrum with $\chi^{2}$ $/$ dof $=1582 / 426$. Therefore, we conclude that this SNR is dominated by IPs.

\subsection{DEM L71}

DEM L71 is a middle-aged, shell-like SNR where a doubleshock morphology was observed by Chandra observations (Hughes et al. 2003), suggesting the presence of both reverse- shocked ejecta and swept-up ISM. Hughes et al. (2003) also revealed that the X-ray spectra from the interior regions are dominated by strong Fe L emission. Similar to the spectral analysis of N49B, we fit the XIS spectrum of DEM L71 with a two-component IP model consisting of a low- $k T_{\mathrm{e}}$ ISM and a high- $k T_{\mathrm{e}}$ ejecta. The fit is acceptable with $\chi^{2} /$ dof of $291 / 229$ as is given in Table 2. The Fe abundance is highest among the four SNRs, consistent with previous studies (e.g., Hughes et al. 1998, 2003) where the Type Ia origin of this SNR was suggested. If we assume $k T_{\text {init }}>k T_{\mathrm{e}}$, then an unacceptable fit $\left(\chi^{2} /\right.$ dof $\left.=399 / 228\right)$ is obtained, ruling out an RP scenario for this remnant.

\section{DISCUSSION}

\subsection{Plasma Conditions in the Observed SNRs}

We have systematically analyzed X-ray spectra from the four LMC SNRs (N49, N23, N49B, and DEM L71) and revealed robust evidence for overionization in N49-this is the first discovery of an RP from an extragalactic SNR. The other SNRs, including N23 from which the presence of an RP was previously reported (Broersen et al. 2011), can be fairly well characterized by an IP or a nearly CIE plasma.

The previous claim of the recombining state in N23 was based on the enhanced $G$-ratio of the $\mathrm{O}$ VII lines measured using XMM-Newton RGS. Although Broersen et al. (2011) claimed that the broadband spectrum of XMM-Newton MOS can also be reproduced by an RP model, their best-fit model is clearly ruled out by our analysis of the XIS spectrum with better photon statistics. Since our analysis excluded the O vII emission, it is still possible that the swept-up ISM (low- $k T_{\mathrm{e}}$ component) is partially recombining. We note, however, that there are several other processes that can enhance the $G$-ratio: resonance scattering and charge exchange. Notably, a similar $G$-ratio enhancement in the $\mathrm{O}$ VII lines was observed in DEM L71, and was indeed interpreted to be a consequence of resonance scattering (van der Heyden et al. 2003). Since the charge 

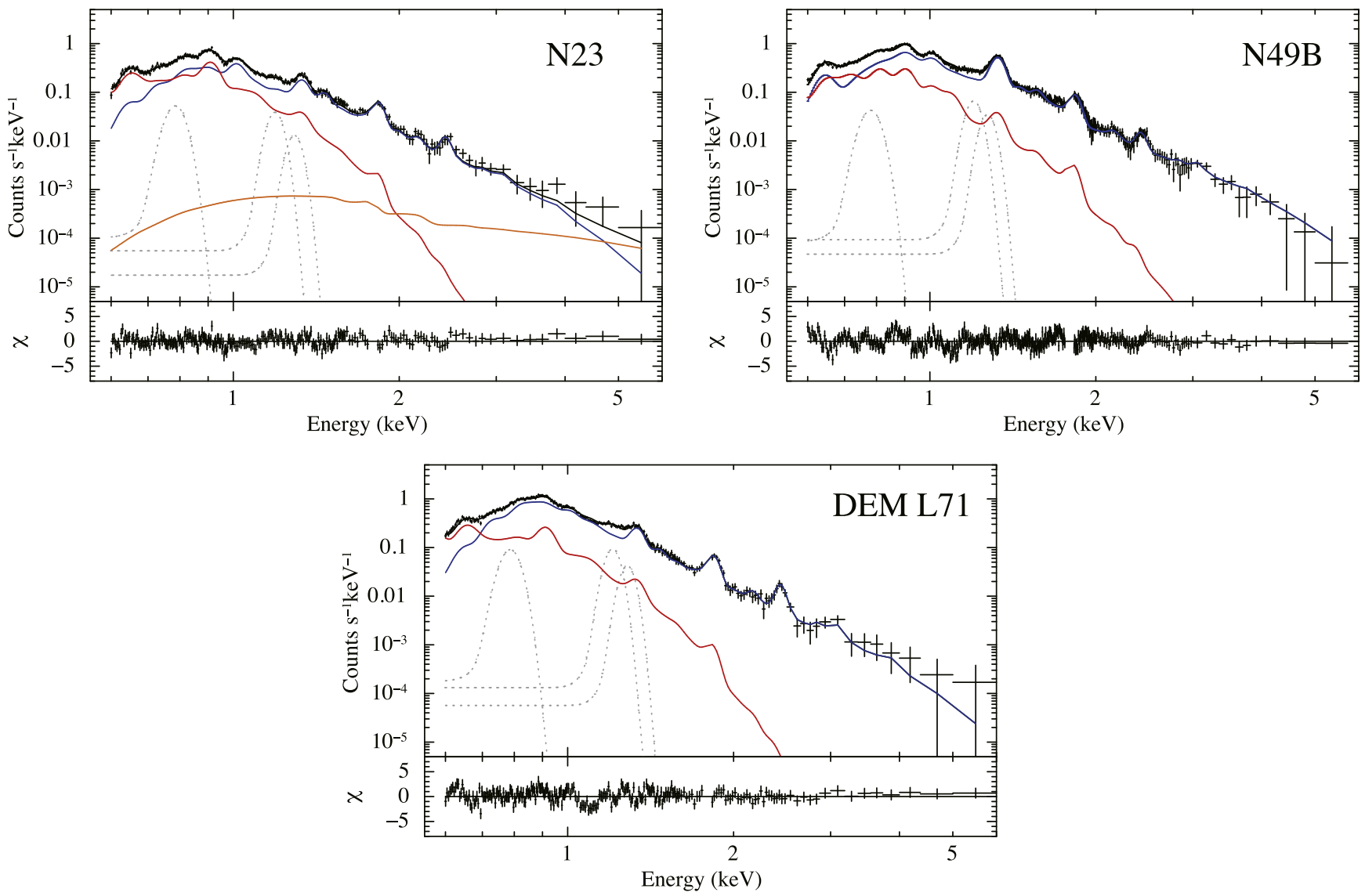

Figure 4. XIS FI spectra of N23 (upper left), N49B (upper right), and DEM L71 (bottom). The best-fit models are overlaid with the black solid lines. The solid blue and red lines represent the best-fit high- $k T_{\mathrm{e}}$ and the low- $k T_{\mathrm{e}}$ components, respectively. The dotted lines show Gaussians for the Fe $\mathrm{L}$ emission missing from the plasma code we used. The orange line in the model of N23 shows the power-law component for CXOU J050552.3-680141 (see the text).

Table 3

Summary of the Four LMC SNRs Studied in This Work

\begin{tabular}{llccccc}
\hline \hline SNR & RP & Age $\left(10^{4}\right.$ years $)$ & SN Type & Morphology & Cloud Interaction \\
\hline N49 & Yes & $0.4-0.5$ & Core-collapse & MM & Yes $($ CO and H $\alpha)$ & Th \\
N23 & No & 0.4 & Core-collapse & MM & Nork, $1,2,3$ \\
N49B & No & 1.09 & Core-collapse & Shell & No \\
DEM L71 & No & $0.4-0.5$ & Ia & Shell & No & $6,7,8$ \\
\hline
\end{tabular}

References. (1) Park et al. (2012), (2) Banas et al. (1997), (3) Melnik \& Copetti (2013), (4) Broersen et al. (2011), (5) Park et al. (2003b), (6) Ghavamian et al. (2003), (7) Hughes et al. (1998), (8) Hughes et al. (2003).

exchange process seems to work in some evolved SNRs (e.g., Puppis A; Katsuda et al. 2012), this process may also be responsible for the high $G$-ratio observed in N23.

\subsection{Origin of the $R P$}

We summarize the characteristics of the four SNRs studied in this work in Table 3. Only N49 is interacting with dense clouds identified by the CO (Banas et al. 1997) and $\mathrm{H} \alpha$ (Melnik \& Copetti 2013) observations. Given that similar cloud interaction is observed in most SNRs from which the presence of an RP has been confirmed (e.g., W49B; Ozawa et al. 2009), thermal conduction into surrounding clouds might play an important role in forming the RP (see Zhou et al. 2011, for a discussion). In this scenario, there should be a spatial correlation between the electron temperature of the SNR plasma and the cloud density. Future deep observations of N49 with better spatial resolution are necessary to assess this possibility.

An alternative is that the adiabatic expansion of the SNR caused a rapid cooling of electrons and the resulting recombining state of the plasma. In this scenario, the SN ejecta should interact with dense circumstellar matter (CSM) in the early phase of the SNR evolution, and the highly ionized ejecta and CSM expand drastically after the SNR blast wave breaks out to the low-density ISM (Itoh \& Masai 1989). Therefore, the ionization timescale $\left(n_{\mathrm{e}} t\right)$ in this scenario is characterized by the elapsed time and the density evolution history since the break out took place. To evaluate the possibility of this scenario, we estimate the electron density in the present RP of $\mathrm{N} 49$ using the derived $\mathrm{EM}\left(=n_{\mathrm{e}} n_{\mathrm{H}} V\right)$. Since the plasma density is known to be highly inhomogeneous in this remnant (Park et al. 2012), we analyze archival Chandra data of N49 to investigate the surface brightness profile. We 
Table 4

List of the RP SNRs in Order of Increasing Electron Temperature

\begin{tabular}{|c|c|c|c|c|c|c|c|}
\hline SNR & $\begin{array}{c}\text { Age } \\
\left(10^{4} \text { years }\right)\end{array}$ & $\begin{array}{l}k T_{\text {init }} \\
(\mathrm{keV})\end{array}$ & $\begin{array}{c}k T_{\mathrm{e}} \\
(\mathrm{keV})\end{array}$ & $\begin{array}{c}n_{\mathrm{e}} t \\
\left(10^{11} \mathrm{~cm}^{-3} \mathrm{~s}\right)\end{array}$ & Cloud Interaction & Compact Object & References \\
\hline G359.1-0.5 & $>1$ & $0.87_{-0.11}^{+0.15}$ & $0.29 \pm 0.02$ & $<4.42$ & Yes & $\ldots$ & $1,2,24$ \\
\hline G346.6-0.2 & $\ldots$ & 5 (fixed) & $0.30_{-0.01}^{+0.03}$ & $4.8_{-0.4}^{+0.1}$ & Yes & $\ldots$ & 3,25 \\
\hline W28 & $\sim 4$ & 3 (fixed) & $0.40_{-0.03}^{+0.02}$ & 6.31 & Yes & PSR $1758-23$ & $4,5,6,24$ \\
\hline W44 & $\sim 2$ & $1.07_{-0.06}^{+0.08}$ & $0.48 \pm 0.02$ & $6.76 \pm 0.5$ & Yes & PSR B1853+01 & $7,8,9,24$ \\
\hline СТВ $37 \mathrm{~A}$ & $\sim 1$ & 5 (fixed) & $0.49_{-0.06}^{+0.09}$ & $13_{-1}^{+3}$ & Yes & CXOU J171419.8-383023 & $10,11,12,24$ \\
\hline 3C391 & $\ldots$ & $1.8_{-0.6}^{+1.6}$ & $0.495 \pm 0.015$ & $14.0_{-2.2}^{+1.5}$ & Yes & $\ldots$ & 13,24 \\
\hline MSH $11-61$ A & $1-2$ & 5 (fixed) & $0.513_{-0.003}^{+0.004}$ & $12.2 \pm 0.4$ & Possible & IGR J11014-6103 & $14,15,16,26$ \\
\hline N49 & $0.4-0.5$ & $11 \pm 1$ & $0.62 \pm 0.01$ & $7.00_{-0.02}^{+0.44}$ & Yes & SGR 0526-66 & This work, 17 \\
\hline IC 443 & $\sim 0.4$ & 10 (fixed) & $0.65 \pm 0.04$ & $9.8 \pm 1.1$ & Yes & XMMU J061804.3+222732 & $18,19,20,21,24$ \\
\hline W49B & $\sim 0.4$ & $\ldots$ & $1.52_{-0.02}^{+0.01}$ & $\ldots$ & Yes & $\ldots$ & $22,23,27$ \\
\hline
\end{tabular}

Note. The plasma parameters for IC 443 and W49B were determined only from a high-energy band around Fe-K lines (e.g., Ozawa et al. 2009; Ohnishi et al. 2014), while those for the other SNRs were determined from intermediate-mass elements.

References. (1) Aharonian et al. (2008b), (2) Ohnishi et al. (2011), (3) Yamauchi et al. (2013), (4) Rho \& Borkowski (2002), (5) Sawada \& Koyama (2012), (6) "runaway" Pulsar; Frail et al. (1993), (7) Cox et al. (1999), (8) Uchida et al. (2012), (9) Pulsar plus Pulsar Wind Nebula; Petre et al. (2002), (10) Yamauchi et al. (2014), (11) Wolszczan et al. (1991), (12) Pulsar Wind Nebula candidate; Aharonian et al. (2008a), (13) Sato et al. (2014), (14) Slane et al. (2002), (15) Kamitsukasa et al. (2015), (16) Pulsar plus Pulsar Wind Nebula; Pavan et al. (2014), (17) Park et al. (2012), (18) Lee et al. (2008), (19) Yamaguchi et al. (2009), (20) Ohnishi et al. (2014), (21) Neutronstar plus Synchrotron Nebula; Olbert et al. (2001), (22) Hwang et al. (2000), (23) Ozawa et al. (2009), (24) Hewitt et al. (2008), (25) Koralesky et al. (1998), (26) Filipović et al. (2005), (27) Keohane et al. (2007).

find that $\sim 50 \%$ of the photon flux in the $\mathrm{Si}$ band $(1.7-2.1 \mathrm{keV})$ is coming from the brightest southeast region (approximately a 0 . $4 \times 0.25 \times 0.25$ ellipsoid), and the other $\sim 50 \%$ is from the remaining faint region in the entire SNR (a sphere with a radius of 0.6 ). Therefore, the volumes and the electron densities are estimated to be $9.6 \times 10^{57}$ and $1.0 \mathrm{~cm}^{-3}$ for the bright region, and $7.3 \times 10^{58}$ and $0.38 \mathrm{~cm}^{-3}$ for the faint region at a distance of $50 \mathrm{kpc}$. If we simply divide the best-fit $n_{\mathrm{e}} t$ in the ejecta component $\left(7.0 \times 10^{11} \mathrm{~cm}^{-3} \mathrm{~s}\right)$ by the derived electron density, then we obtain plasma ages of 22-58 kyr, more than a few times larger than the dynamical age of N49 ( 4800 years; Park et al. 2012). This is not surprising because the plasma must have had a higher electron density in the past. Yamaguchi et al. (2012) also obtained a similar result from the Suzaku observation of IC 443 where the plasma age estimate $(\sim 11 \mathrm{kyr})$ is significantly higher than the SNR age of $\sim 4000$ years (Troja et al. 2008).

In Table 4, we summarize the physical properties of the RP SNRs identified so far. While the estimated ages roughly correlate with $k T_{\mathrm{e}}$, the values of $n_{\mathrm{e}} t$ show no clear trend. This may be partly due to the technical reason that the parameter $n_{\mathrm{e}} t$ is coupled to that of $k T_{\text {init }}$ in the spectral fitting. Nevertheless, Table 4 shows that the $n_{\mathrm{e}} t$ values exceed $\sim 10^{11} \mathrm{~cm}^{-3} \mathrm{~s}$ in all SNRs, implying that the overionization did not start from the last few hundred years, but from the early epoch in the SNR. This fact likely favors the adiabatic cooling scenario for most RP SNRs.

\subsection{Progenitor of N49 and SGR 0526-66}

The SN type of N49 has been controversial. Park et al. (2012) suggested a Type Ia origin based on the $\mathrm{Si} / \mathrm{S}$ abundance ratio in the ejecta measured by the Chandra data. On the other hand, the environment of N49, i.e., a nearby OB association (Chu \& Kennicutt 1988) and young stellar clusters (Klose et al. 2004), are more common in core-collapse SNRs. A recent systematic study of $\mathrm{Fe} \mathrm{K}$ emission in young and middle-aged SNRs also agrees on a core-collapse origin (Yamaguchi et al. 2014a). Given that an RP has never been observed in a Type Ia
SNR, our results strongly support a core-collapse scenario for this SNR. It should also be noted that the formation of an RP requires dense CSM, which is only made by a massive progenitor with a significant mass loss rate (Moriya 2012; Shimizu et al. 2012). The total mass of the RP component is roughly estimated to be $\sim 26 M_{\odot}$ using the density and emitting volume derived in Section 4.2, which is in marginal agreement with the progenitor mass of $12.5-21.5 M_{\odot}$ constrained from the local star formation history around N49 (Badenes et al. 2009).

Since a core-collapse origin is strongly suggested for N49, the compact object SGR 0526-66, which is a known magnetar candidate (e.g., Park et al. 2012), might be associated with the SNR. The large distance between SGR 0526-66 and the geometric center of N49 requires a high kick velocity $\left(\sim 1100 \mathrm{~km} \mathrm{~s}^{-1}\right)$ for the SGR at an SNR age of $\sim 4800$ years, and hence Park et al. (2012) argued against the physical association between these objects. However, the theoretical prediction is that a magnetorotational core-collapse $\mathrm{SN}$ explodes asymmetrically with bipolar jets and produces a magnetar with a large kick velocity up to $\sim 1000 \mathrm{~km} \mathrm{~s}^{-1}$ (Sawai et al. 2008). Moreover, there are several compact objects located extremely off-center from the associated RP SNRs. For instance, IGR J11014-6103 is located outside the shell of MSH 11-61 A. Pavan et al. (2014) indicated that this neutron star is escaping from MSH 11-61 A at a velocity exceeding $1000 \mathrm{~km} \mathrm{~s}^{-1}$. Also, PSR 1758-23 is known as a "runaway pulsar" which might be physically associated with W28 (Frail et al. 1993). Two other compact objects, CXOU J171419.8-383023 in CTB 37 A (Aharonian et al. 2008a) and XMMU J061804.3+222732 in IC 443 (Olbert et al. 2001), are also found far from the geometric center of their host SNRs. Taking these facts into consideration, we propose that SGR 0526-66 is the plausible candidate for the compact remnant of N49.

\section{CONCLUSIONS}

We have shown clear evidence for an RP in the SNR N49, which is the first report of the robust detection of an RP from an 
extra-galactic SNR achieved by the high sensitivity and good energy resolution of the XIS on board Suzaku. The other SNRs, including N23 where the presence of an RP was previously claimed (Broersen et al. 2011), can be well characterized by an IP or nearly CIE plasma. Future observation of N49 using highresolution X-ray spectrometers, like ASTRO-H (Takahashi et al. 2014), is highly encouraged; our Suzaku result predicts the detection of the Fe RRC as well as enhanced forbidden lines due to recombination processes (see Section 3.1). We also emphasize the importance of a systematic study of RP SNRs, which will help us to understand the physical processes responsible for the formation of the RPs in more detail.

The authors thank Dr. T. G. Tsuru for a careful reading of our manuscript. This work is supported by JSPS KAKENHI grant Nos. 26800102 (H.U.) and 2450229 (K.K.).

Facility: Suzaku (XIS).

\section{REFERENCES}

Aharonian, F., Akhperjanian, A. G., Barres de Almeida, U., et al. 2008a, A\&A, 490,685

Aharonian, F., Akhperjanian, A. G., Barres de Almeida, U., et al. 2008b, A\&A, 483, 509

Anders, E., \& Grevesse, N. 1989, GeCoA, 53, 197

Badenes, C., Harris, J., Zaritsky, D., \& Prieto, J. L. 2009, ApJ, 700, 727

Banas, K. R., Hughes, J. P., Bronfman, L., \& Nyman, L.-A 1997, ApJ, 480, 607

Brickhouse, N. S., Dupree, A. K., Edgar, R. J., et al. 2000, ApJ, 530, 387

Broersen, S., Vink, J., Kaastra, J., \& Raymond, J. 2011, A\&A, 535, A11

Chu, Y.-H., \& Kennicutt, R. C., Jr. 1988, AJ, 96, 1874

Cline, T. L., Desai, U. D., Teegarden, B. J., et al. 1982, ApJL, 255, L45

Cox, D. P., Shelton, R. L., Maciejewski, W., et al. 1999, ApJ, 524, 179

Dickel, J. R., Chu, Y.-H., Gelino, C., et al. 1995, ApJ, 448, 623

Dickel, J. R., \& Milne, D. K. 1998, AJ, 115, 1057

Dickey, J. M., \& Lockman, F. J. 1990, ARA\&A, 28, 215

Feast, M. 1999, PASP, 111, 775

Filipović, M. D., Payne, J. L., Reid, W., et al. 2005, MNRAS, 364, 217

Frail, D. A., Kulkarni, S. R., \& Vasisht, G. 1993, Natur, 365, 136

Gaensler, B. M., Slane, P. O., Gotthelf, E. V., \& Vasisht, G. 2001, ApJ, 559, 963

Ghavamian, P., Rakowski, C. E., Hughes, J. P., \& Williams, T. B. 2003, ApJ, 590,833

Gu, M. F., Chen, H., Brown, G. V., Beiersdorfer, P., \& Kahn, S. M. 2007, ApJ, 670,1504

Hayato, A., Bamba, A., Tamagawa, T., \& Kawabata, K. 2006, ApJ, 653, 280

Hewitt, J. W., Yusef-Zadeh, F., \& Wardle, M. 2008, ApJ, 683, 189

Hughes, J. P., Ghavamian, P., Rakowski, C. E., \& Slane, P. O. 2003, ApJL, 582, L95

Hughes, J. P., Hayashi, I., \& Koyama, K. 1998, ApJ, 505, 732

Hwang, U., Petre, R., \& Hughes, J. P. 2000, ApJ, 532, 970

Ishisaki, Y., Maeda, Y., Fujimoto, R., et al. 2007, PASJ, 59, 113

Itoh, H., \& Masai, K. 1989, MNRAS, 236, 885

Jones, T. W., Rudnick, L., Jun, B.-I., et al. 1998, PASP, 110, 125
Kaastra, J. S., Mewe, R., \& Nieuwenhuijzen, H. 1996, in the 11th Coloquium on UV and X-ray Spectroscopy of Astrophysical and Laboratory Plasmas, ed. K. Yamashita \& T. Watanabe, 411

Kamitsukasa, F., Koyama, K., Uchida, H., et al. 2015, PASJ, 67, 16

Katsuda, S., Tsunemi, H., Mori, K., et al. 2012, ApJ, 756, 49

Kawasaki, M., Ozaki, M., Nagase, F., Inoue, H., \& Petre, R. 2005, ApJ, 631, 935

Kawasaki, M. T., Ozaki, M., Nagase, F., et al. 2002, ApJ, 572, 897

Keohane, J. W., Reach, W. T., Rho, J., \& Jarrett, T. H. 2007, ApJ, 654, 938

Klose, S., Henden, A. A., Geppert, U., et al. 2004, ApJL, 609, L13

Koralesky, B., Frail, D. A., Goss, W. M., Claussen, M. J., \& Green, A. J. 1998, AJ, 116, 1323

Koyama, K., Tsunemi, H., Dotani, T., et al. 2007, PASJ, 59, 23

Lee, J.-J., Koo, B.-C., Yun, M. S., et al. 2008, AJ, 135, 796

Long, K. S., Helfand, D. J., \& Grabelsky, D. A. 1981, ApJ, 248, 925

Masai, K. 1984, Ap\&SS, 98, 367

Melnik, I. A. C., \& Copetti, M. V. F. 2013, A\&A, 553, A104

Mitsuda, K., Bautz, M., Inoue, H., et al. 2007, PASJ, 59, 1

Moriya, T. J. 2012, ApJL, 750, L13

Nakashima, S., Nobukawa, M., Uchida, H., et al. 2013, ApJ, 773, 20

Ohnishi, T., Koyama, K., Tsuru, T. G., et al. 2011, PASJ, 63, 527

Ohnishi, T., Uchida, H., Tsuru, T. G., et al. 2014, ApJ, 784, 74

Olbert, C. M., Clearfield, C. R., Williams, N. E., Keohane, J. W., \& Frail, D. A. 2001, ApJL, 554, L205

Ozawa, M., Koyama, K., Yamaguchi, H., Masai, K., \& Tamagawa, T. 2009, ApJL, 706, L71

Park, S., Burrows, D. N., Garmire, G. P., et al. 2003a, ApJ, 586, 210

Park, S., Hughes, J. P., Slane, P. O., et al. 2012, ApJ, 748, 117

Park, S., Hughes, J. P., Slane, P. O., et al. 2003b, ApJL, 592, L41

Pavan, L., Bordas, P., Pühlhofer, G., et al. 2014, A\&A, 562, A122

Petre, R., Kuntz, K. D., \& Shelton, R. L. 2002, ApJ, 579, 404

Petruk, O. 2001, A\&A, 371, 267

Rho, J., \& Borkowski, K. J. 2002, ApJ, 575, 201

Rho, J., \& Petre, R. 1998, ApJL, 503, L167

Russell, S. C., \& Dopita, M. A. 1992, ApJ, 384, 508

Sato, T., Koyama, K., Takahashi, T., Odaka, H., \& Nakashima, S. 2014, PASJ, 66, 124

Sawada, M., \& Koyama, K. 2012, PASJ, 64, 81

Sawai, H., Kotake, K., \& Yamada, S. 2008, ApJ, 672, 465

Shimizu, T., Masai, K., \& Koyama, K. 2012, PASJ, 64, 24

Slane, P., Smith, R. K., Hughes, J. P., \& Petre, R. 2002, ApJ, 564, 284

Takahashi, T., Mitsuda, K., Kelley, R., et al. 2014, Proc. SPIE, 9144, 25

Tawa, N., Hayashida, K., Nagai, M., et al. 2008, PASJ, 60, 11

Troja, E., Bocchino, F., Miceli, M., \& Reale, F. 2008, A\&A, 485, 777

Uchida, H., Koyama, K., Yamaguchi, H., et al. 2012, PASJ, 64, 141

Uchida, H., Yamaguchi, H., \& Koyama, K. 2013, ApJ, 771, 56

van der Heyden, K. J., Bleeker, J. A. M., Kaastra, J. S., \& Vink, J. 2003, A\&A, 406, 141

Vancura, O., Blair, W. P., Long, K. S., \& Raymond, J. C. 1992, ApJ, 394, 158 White, R. L., \& Long, K. S. 1991, ApJ, 373, 543

Wolszczan, A., Cordes, J. M., \& Dewey, R. J. 1991, ApJL, 372, L99

Yamaguchi, H., Badenes, C., Petre, R., et al. 2014a, ApJL, 785, L27

Yamaguchi, H., Eriksen, K. A., Badenes, C., et al. 2014b, ApJ, 780, 136

Yamauchi, S., Minami, S., Ota, N., \& Koyama, K. 2014, PASJ, 66, 2

Yamauchi, S., Nobukawa, M., Koyama, K., \& Yonemori, M. 2013, PASJ, 65,6

Yamaguchi, H., Ozawa, M., Koyama, K., et al. 2009, ApJL, 705, L6

Yamaguchi, H., Ozawa, M., \& Ohnishi, T. 2012, AdSpR, 49, 451

Zhou, X., Miceli, M., Bocchino, F., Orlando, S., \& Chen, Y. 2011, MNRAS, 415,244 